\title{
Construction and Simulation of a Strategic HR Decision Model Based on Recurrent Neural Network
}

\author{
Xiaorong Li, ${ }^{1}$ Lijun Zhang $\left(\mathbb{D},{ }^{2}\right.$ Dongchen $\mathrm{Li}^{3}{ }^{3}$ and Dan Guo ${ }^{4}$ \\ ${ }^{1}$ Shool of Faculty of Management and Economics, Kunming University of Science and Technology, Kunming, \\ Yunnan 650000, China \\ ${ }^{2}$ School of Management, Hebei GEO University, Shijiazhuang, Hebei 050031, China \\ ${ }^{3}$ School of Management Engineering and Business, Hebei University of Engineering, Handan, Hebei 056038, China \\ ${ }^{4}$ School of Earth and Engineering, Hebei University of Engineering, Handan, Hebei 056038, China \\ Correspondence should be addressed to Lijun Zhang; zljj14619@hgu.edu.cn
}

Received 5 November 2021; Accepted 16 December 2021; Published 4 January 2022

Academic Editor: Miaochao Chen

Copyright (c) 2022 Xiaorong Li et al. This is an open access article distributed under the Creative Commons Attribution License, which permits unrestricted use, distribution, and reproduction in any medium, provided the original work is properly cited.

In this paper, RNN (Recurrent Neural Network) algorithm is used to conduct an in-depth analysis of HR strategic decisionmaking and an HR strategic decision model is constructed for simulation. The four evaluation dimensions of index screening are extracted, the generalized gray correlation analysis is applied to screen the initial selection indexes of HR strategic decisionmaking, and then the HR strategic decision-making index system of power supply enterprises is constructed. On this basis, the applicability of the BP neural network in HR strategic decision-making is analysed and demonstrated, a BP neural network-based HR strategic decision-making model for power supply enterprises is constructed, and the rationality of the model is illustrated through the model training and testing. Finally, an empirical study is conducted with $\mathrm{S}$ power supply company as an example to illustrate the operability of the BP network model for HR strategic decision-making. The results of this paper provide a scientific basis for human resource decision-making in power supply enterprises and provide theoretical support for promoting the healthy development of power supply enterprises. Improving the level of human resource management can make fuller use of human resources and realize the strategic goals of the enterprise. A strategy for optimizing the training of hybrid convolutional neural networks is proposed, using an exponential linear unit activation function to solve the problem of neuron dead zones, a hybrid pooling strategy is used to improve the problem of feature information loss in maximum pooling processing, and an improved cross-entropy loss function is used to solve the problem of insufficient learning of difficult classification samples. The optimization of the model training process is finally completed, and the recommendation quality is improved. The Bayesian probability distribution table is learned to be filled in the expert data set; finally, the accuracy and effectiveness of the model are tested on the experimental platform. The results of the experiments show that the established Bayesian model beats the platform's built-in intelligent method with a $78.2 \%$ win rate. That is, the model can make intelligent recommendations for strategies in staffed and unmanned platform decision-making and command-and-control combat units to execute tactical actions to achieve the best operational effectiveness.

\section{Introduction}

Human resource strategy has a supportive role in the realization of business development strategies, and with the development of the economy, human resource strategy has received a great deal of attention from a wide range of scholars and business managers. The complexity and variability of the environment have gradually led to the understanding that competition in all socioeconomic activities has changed into competition for human resources [1]. Only highly competitive human resources can provide support for the realization of the strategic goals of enterprises, enable enterprises to gain competitive advantages in the complex and changing competitive environment, promote enterprise development, and maximize enterprise profits. Modern human resource strategy pays more 
attention to the strategic position of human resources in the enterprise, and talent quality and workforce development are regarded as holistic concepts, so it seems that making good strategic human resource decisions becomes an important thing for the enterprise. This paper introduces artificial neural networks into HR strategic decision-making, which will enrich HRM theory and expand the application field of artificial neural network methods [2]. At the same time, it also provides a reasonable and operable tool for power supply enterprises to make HR strategic decisions more scientifically and effectively improve HRM and can make fuller use of human resources to achieve the strategic goals of enterprises. In the field of computers, data mining technology is a hot research topic, and time-series analysis and prediction technology are important branches of data mining. Time series refers to a series of observations obtained in the process of production and scientific research and so on, recorded in chronological order, which is a multiple variable at different moments on the formation of random data, reflecting the development of phenomena that change the law [3]. Time series has three main characteristics: randomness, continuity, and periodicity. Data at the current point in time can be disturbed by numerous external influences, so the time series is random.

In terms of fine-grained time, the values of adjacent time series are continuous and time-dependent and therefore have continuity. That is, the data at the current point in time is highly likely to be influenced by a previous point in time or multiple previous points in time, called short-term dependence and long-term dependence, respectively. Periodicity refers to the phenomenon that, taken as a whole, time-series data tend to exhibit periodic changes or some sort of trend. The above three characteristics illustrate both the feasibility and the difficulty of predicting time series. Therefore, time series is of high research significance. Time-series forecasting models have a wide range of applications, with significant application scenarios in areas such as electricity demand forecasting, financial market forecasting, sales performance forecasting, environmental weather forecasting, and medical diagnosis. Accurate stock market forecasting can bring huge profit gains; accurate weather forecasting can avoid disaster risks; accurate electricity demand forecasting can avoid energy waste and ensure service quality [4]. Whether for commercial gain or risk avoidance, improving the accuracy of time-series forecasting has great research value and practical value. In the overall development strategy of an enterprise, human resource strategy occupies an important position as a substrategy. The success or failure of HR strategy formulation has a great impact on the overall strategy of the enterprise. With the development of human society, people increasingly realize that the competition of talents is the core of enterprise competition, and human resource strategy has become the content of enterprise concern [5]. With rapid economic development and rapid changes in the internal and external environment faced by enterprises, enterprises must be able to respond quickly to environmental changes, make timely responses, and make quick decisions. It is the random data formed by a certain variable or multiple variables at different moments, reflecting the development and change law of the phenomenon: randomness, continuity, and periodicity. The data at the current time point will be disturbed by many external factors, so the time series is random.

In the context of information explosion and globalization, the whole world is included in the flood of the knowledge economy, and mankind is in a new era; in such a new era of rapid expansion of knowledge, science, and technology, the strategic resources and main driving force of economic and social development have shifted to culture knowledge and science and technology, and the implicit human capital which unites knowledge skills and intellectual factors has become the most important strategic capital. After long-term accumulation, some special human capital has become an important core resource of the enterprise, with assets of heterogeneous characteristics of the enterprise. This is also the source of the core competitiveness of the enterprise. From the current research status of implicit human capital, we can see that this research field is still in the primary development stage. Although there is no authoritative theoretical system and applicable model, the study of implicit human capital as the core value represents the new theoretical mainstream. As seen from the status of research, the study of intellectual capital lags foreign research, but the study of tacit human capital is in the leading stage. As in the primary stage of socialism, influenced by the semifeudal and semicolonial history, the history of the emergence and development of enterprises is relatively short, and the play of the role of implicit human capital possessed by individuals and organizations within enterprises has an immeasurable impact on enhancing the core competitiveness of enterprises. Therefore, the national policy indicators will be retained, and the market competition indicators will be deleted. Looking at the overall situation of employee turnover rate, it is basically maintained at a relatively reasonable level and is lower than the industry average.

\section{Related Works}

In terms of human resource management ideas and human resource management models, the theoretical results of strategic human resource management based on national conditions are presented, and the formulation and selection of human resource strategies are thoroughly analysed and discussed, drawing on foreign research results. The problems of (strategic) human resource management are analysed, and the current research in this field of strategic human resource management is sorted out, starting from identifying the meaning of strategic human resource management, putting forward the problems in theoretical construction and the selection and measurement of research objects, and making an in-depth analysis of possible future research directions [6]. A model of HR strategy improvement based on GREP is proposed; a model of HR strategy based on job qualifications is proposed [7]. So far, on the definition of $\mathrm{HR}$ strategy, scholars have different understandings and different emphasis, some focus on planning, and some focus on strategic positioning, but their understandings have their commonality, they have the same understanding of the 
essence of HR, and they believe that HR strategy has three levels of meaning; namely, firstly, the formulation of HR strategy must be based on basic judgments; secondly HR strategy has a guiding significance to the direction of HRM; third is that HR strategy should be formulated following its guiding ideology, clarify the main objective of the strategy, and develop corresponding measures to achieve this main objective [8]. Regarding the research on the method of HR strategy decision, most of the scholars' researches focus on different aspects of HRM, such as salary decision and planning decision, while there are fewer researches on the decision of overall HR strategy and the method of selecting an HR strategy, and most of the proposed strategic decision methods are also subjective and lacking in quantitative methods [9].

For the analysis of time-series data, in the beginning, statistical learning methods were used to analyse the time series according to their characteristics. Later, machine learning methods continued to develop, and researchers at home and abroad gradually used to introduce machine learning methods into the study of time series. Later, with the emergence of neural network methods, the use of neural networks to perform time-series analysis became popular [10]. With the continuous improvement of computer hardware levels, neural network models with more complex structures and stronger expression capabilities emerged. The analysis of time series by neural network models with multiple hidden layers, that is, deep neural network models, has continued to develop and become popular for scholars' research [11]. At the same time, these research methods are being developed in conjunction with expertise in their respective fields, making it necessary for scholars to research with expertise in the corresponding research areas [12]. In summary, the current time-series prediction methods mainly include statistical learning methods, traditional machine learning methods, feedforward neural network methods, Recurrent Neural Networks, and their variants methods, and this section will introduce the domestic and international research history and development status of each method one by one [13]. The neural network language model, as one of the application scenarios of the neural network model, has been successful in the fields of speech recognition, natural language processing, and so on.

It is proposed that human resource allocation is a core process in MIS projects. The uncertainty in the demand for professionals as well as experts in individual projects makes it more difficult to allocate personnel in projects. The authors apply a dynamic planning model to allocate human resources. This method considers aspects such as the complexity of each project as well as the existing capabilities and required skills of the project staff. The model is formed by the concepts of constitutive rational attitudes and incrementality and is based on the two dimensions of internal and external labour markets, controlling the quantity and quality of the product's workforce. Four main strategies are introduced in this model, which are paternalistic strategy, committed strategy, free-agent strategy, and auxiliary strategy. It is an HR model that integrates the company's strategy, HR strategy, and OS strategy.

\section{Analysis of RNN for the Strategic Human Resource Decision-Making Model}

3.1. Model Construction of Improved RNN for Strategic HR Decision-Making. The hybrid recommendation algorithm aims to take advantage of both by combining content-based recommendation algorithms and collaborative filtering recommendation algorithms to get better performance in prediction or recommendation [14]. The two recommendation algorithms are run separately, and then the results of both are combined and used to make recommendations. The features obtained from the content-based recommendation algorithm are fused and integrated into the collaborative filtering recommendation algorithm. Integrate the feature fusion obtained by the collaborative filtering recommendation algorithm into the content-based recommendation algorithm. The features obtained from the content-based recommendation algorithm and the collaborative filtering recommendation algorithm are extracted separately, fused, and integrated into a separate reinforcement model. The hybrid recommendation algorithm can complement each other's strengths and use content analysis to solve the coldstart problem of new items or new users with better recommendation performance. However, the selection of the combination methods and the order of the combination need a lot of experiments to find a better combination, and the weight distribution of the recommendation results obtained by different methods also needs to be tested and analysed experimentally. From the current research status of implicit human capital, this research field is still in the initial stage of development. Although there is no authoritative theoretical system and applicable model at present, the research on hidden human capital as the core value represents a new theoretical mainstream.

$$
\begin{aligned}
& \alpha_{d}=\lim _{m \longrightarrow \infty} \sum_{i=1}^{m} v_{i d}^{2} x_{i}^{2}, \\
& \beta_{j}=\lim _{d \longrightarrow \infty} \sum_{h=1}^{d}\left(w_{h d}^{2}-1\right)\left(b_{h}^{3}+1\right) .
\end{aligned}
$$

The focus of this paper is on the research of recommendation algorithm improvement in the field of human resources, in response to the current situation that human resources recommendation algorithm is more traditional and single, by analysing the characteristics of the human resources field, using the convolution neural network and gradient to enhance the technology, such as the tree algorithm for the improvement and implementation, to verify the feasibility of deep learning technology in the field of human resources. This paper takes advantage of the emerging technology to improve the quality of HR recommendation and alleviate the current employment problem of "job mismatch," hoping to provide reference cases for companies engaged in Internet HR recruitment and to raise the attention of industry and academia to HR recommendation research and promote the development of the research field. We also hope to raise the attention of industry and academia on HR recommendation research and 
promote the development of the research field. Therefore, the research topic of this paper has certain technical improvements and social reality significance. The hybrid recommendation algorithm can learn from each other's strengths, uses content analysis to solve the cold-start problem of new projects or new users, and has better recommendation performance. This paper will describe the algorithm in detail in the subsequent contents, and this section will focus on the detailed description of the overall flow of the recommendation algorithm, as shown in Figure 1.

To address the above problems, this paper designs and implements an algorithm applicable to human resource recommendation using gradient boosting tree and convolutional neural network to improve the problem of poor recommendation quality caused by the limited feature extraction ability of traditional recommendation algorithms [15]. The computational efficiency of gradient boosting tree and convolutional neural network can provide a feasibility guarantee for making more diverse recommendations and generate diverse recommendation results by using different job recommendation lengths to make up for the shortcomings of traditional recommendations in a single way. The design idea of the core algorithm in this paper is to first use a gradient boosting tree for feature conversion processing to complete the screening and encoding of the original features, then input the converted features into the hybrid convolutional neural network designed in this paper, and use the high-level feature abstraction learning ability of convolutional processing to obtain a personalized list of human resource recommendation results.

$$
y_{j}^{k}=f\left(\beta_{j}^{2}+\theta_{j}^{2}\right) .
$$

RNN is a type of neural network for processing sequential data. In the previously mentioned feedforward neural networks, information transfer between neurons can only flow in one direction, that is, from the input layer to the output layer, and there are no connections between neurons in the same layer. RNN is more like feedforward neural networks, but it allows backward connections, and connection rights are established between neurons in the same hidden layer.

$$
\begin{aligned}
& s_{t}=f\left(U * x_{t}^{2}-W * s_{t+1}\right), \\
& f_{t}=\delta\left(W_{f} \cdot\left[h_{t}, x_{t}^{2}\right]-b_{f}\right) .
\end{aligned}
$$

The main server executes multiple microbatch processing tasks in parallel to form the corresponding data streams. A memory-based processing approach is used to categorize the data streams according to the source types for unifying the types of data streams, and the categorized data is initially stored to build the initial original data set and provide a data basis for subsequent data preprocessing. In this stream-based distributed data collection scheme, first, multiple data source channels are confirmed with the cooperative enterprise for determining the source types of the collection channels, the data types to be collected are classified as data sources according to the demand type, and then multiple execution threads are allocated to execute data pooling tasks in parallel on the distributed cloud cluster server for receiving data. The subservers dynamically collect different types of data in the form of task queues and then classify and pool different types of data to achieve dynamic real-time updates, as shown in the specific design diagram in Figure 2.

The design idea of the core algorithm is as follows: first use the gradient boosting tree to perform feature conversion processing, complete the screening and coding of the original features, and then input the converted features into the hybrid convolutional neural network designed in this paper. Whenever the subservers complete their respective data aggregation tasks, they break up the streaming computational processing into microbatch processing tasks in the same way as streaming processing. Using microbatch processing time as the criterion, the data is sliced into data streams in a segmented format, and each segment is cached using a memory-based approach. The expert server uses inmemory processing to parallelize the data from multiple segment streams, categorize the data according to their source types, and persist them as raw data sets.

$$
\begin{aligned}
y_{z} & =\Re\left(W_{z} x_{z}-b_{z}\right), \\
\lambda & =\sum_{n}(y \ln p-(1-y) \ln (1+p)) .
\end{aligned}
$$

The data collection scheme used uses a multithreaded approach to perform data collection tasks and improves the processing performance of data collection by operating in a distributed cloud cluster, ensuring throughput and stability of data collection, and providing a certain degree of scalability. The data acquisition adopts a dynamic and direct processing model, unlike the traditional store-and-process model, without first completing data accumulation and landing [16]. A partitioned associative task queue structure is used to improve the throughput rate of data production and consumption, enabling data acquisition tasks to be performed in real time. The use of the in-memory model for storing the collected data reduces the space occupied when local temporary files are saved, thus avoiding the occurrence of data build-up, loss, and so on. At the same time, based on the efficiency of the memory model storage, the data acquisition update is realized by using the task queue, and the data stream is constructed by the time slice division. Using the characteristics of streaming data, the data is operated in parallel directly in memory to speed up the efficiency of processing, improve the processing performance, provide the data basis for subsequent data preprocessing, and ensure that the real-time data collection can be updated.

$$
\begin{aligned}
m_{t} & =\beta_{1} m_{t}-\left(1+\beta_{1}\right) g_{t}, \\
v_{t} & =\beta_{2} m_{t+1}-\left(1-\beta_{1}^{2}\right) g_{t}, \\
\theta_{t} & =\theta_{t-1}+\frac{m_{t}}{\sqrt{v_{t}-m_{t}}} .
\end{aligned}
$$




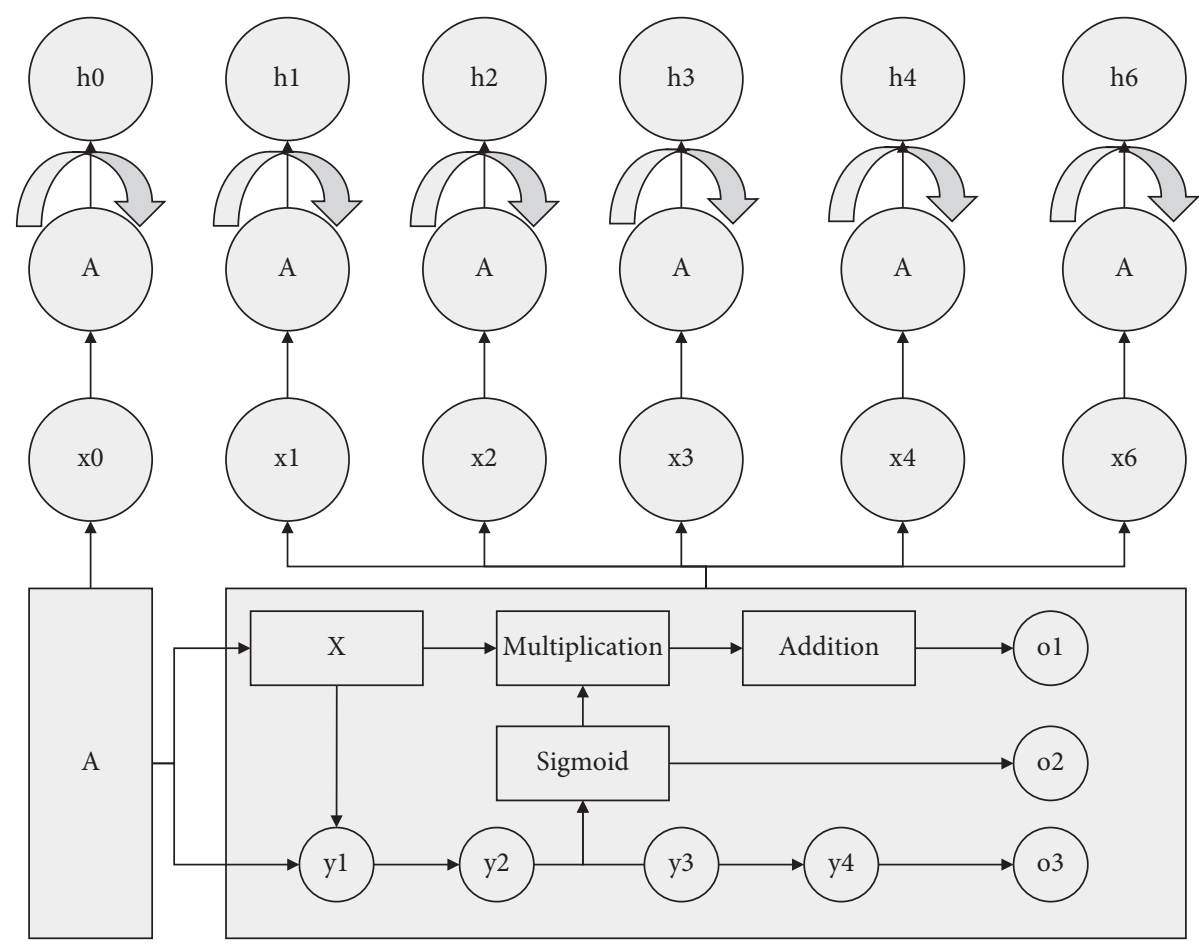

FIgURE 1: Improved RNN framework for strategic HR decision-making.

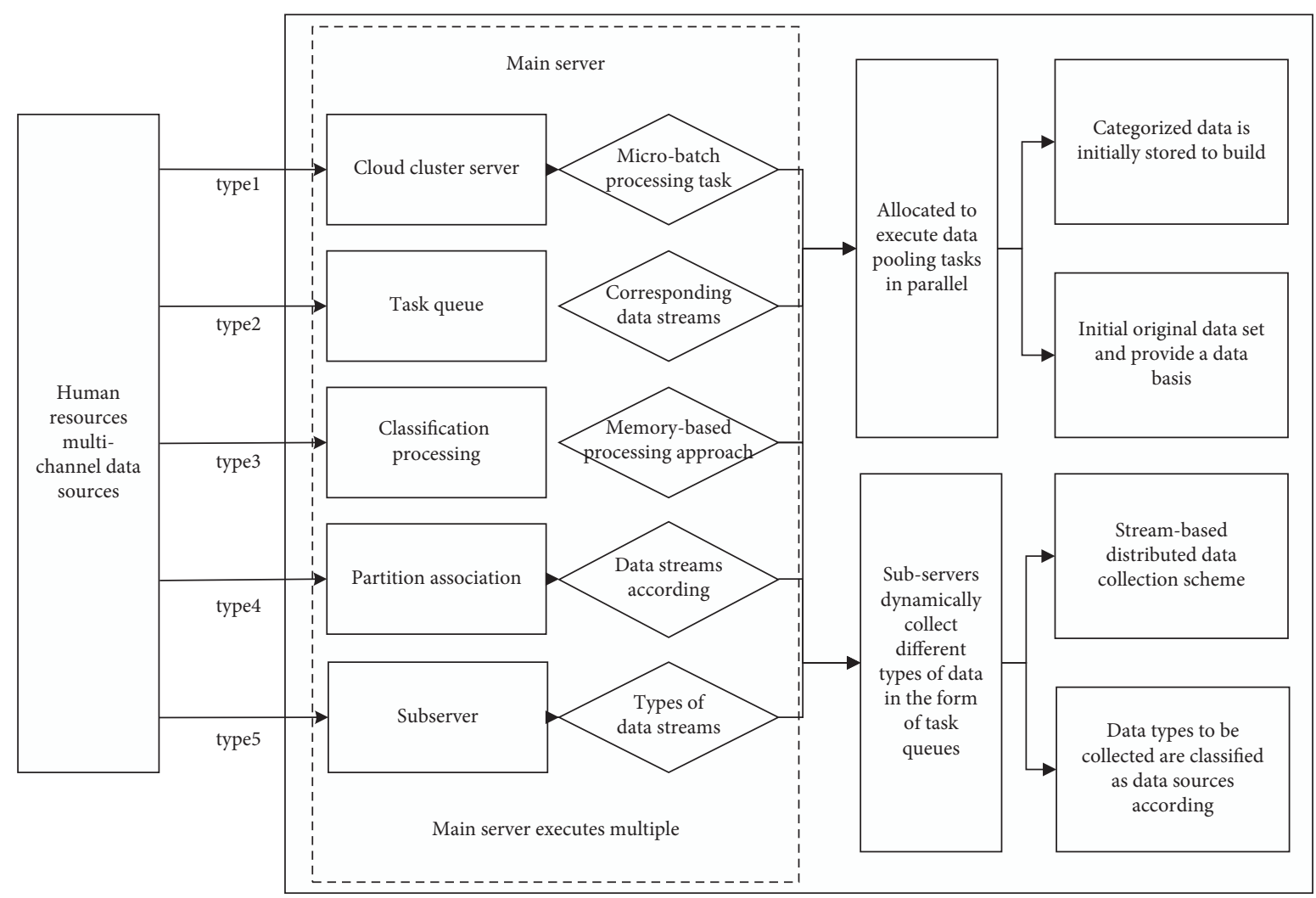

FIGURE 2: Data collection partition task queue construction and data categorization design diagram.

However, in the command-and-control process of manned-unmanned platform collaborative decision-making, the workload of decision-making and control increases drastically due to the increase in the number of unmanned platforms, and the processing capability of the operator cannot meet the requirements of the charge. Therefore, each 
unmanned platform is required to have a certain mission and situational intelligence understanding capability to analyse and extract the key environmental condition information required for operational decision-making embedded in the fused situational map for mission planning based on the high-level mission commands sent by the operator. Both staffed and unmanned platforms can perform decision-making behaviour based on task and posture, with the unmanned platform able to align itself with human behaviour in both explicit and implicit ways, monitoring operator interactions, assessing the operator's cognitive state, and proactively assisting; the operator can understand the behaviour of the autonomous system in some form, analyse its task state, and proactively intervene. The improved decision-making capability of the unmanned platform, which can proactively recommend a strategy based on its understanding of the current situation during the manned-unmanned collaborative decision-making process, will effectively reduce the commander's workload and enable the complementary capabilities of the staffed and unmanned platforms, greatly enhancing the effectiveness of mission execution.

$$
\begin{aligned}
\xi\left(v_{i}(k), v_{j}(k)\right) & =\frac{\Delta \max -p \Delta \min }{\Delta_{i j}(k)-p \Delta \max }, \\
R_{t} & =\frac{1}{s} \sum_{j \neq i} \gamma\left(V_{i}^{2}, V_{j}^{2}\right) .
\end{aligned}
$$

The corporate strategy contains financial strategy, technology strategy, human resource strategy, and so on. This paper stands in the perspective of strategic human resources to study the issue of strategic human resource decision, that is, the planning of human resources in the development of corporate strategy. The whole process of formulating HR strategy should always reflect the concept of enterprise development, and the basis of HR strategy formulation is the analysis of the current HR situation and HR development forecast. The process of formulating HR strategy includes three parts, namely, stating the purpose, confirming the objective, and selecting the strategy [17].

\subsection{Simulation Design of a Strategic HR Decision Model.} The way of screening indicators one by one according to the value of the above single dimension separately belongs to multilevel barrier screening, this method is too harsh screening, and the results of screening may leave only a few indicators, so that the second most important indicators missed, which is not conducive to the realization of the principle of comprehensive construction of the indicator system. For this reason, this paper proposes to use the generalized gray absolute correlation analysis method to comprehensively screen the primary selection indicators. One of the advantages is that the absolute correlation degree has the nature of translation without changing the correlation degree, which can more objectively reflect the degree of correlation between the comparison series and the reference series; the second advantage is that the comprehensive screening is compensatory, while the comprehensive screening based on the primary selection index system is combined screening. In solving important problems, the combined type is better than the multilevel barrier type, the compensatory type is a conclusion that has been proved by theory for a long time, and the comprehensive screening in this paper has the property of combined type screening. Therefore, the selection of the generalized gray absolute correlation method for screening indicators not only screens out the optimal indicators but also screens out the suboptimal indicators with better synthesis, which enhances the rationality of the indicator system. In addition, the gray correlation analysis method requires a low number of samples, at least up to 4 , which is also effective for data without regularity, the calculation results generally do not appear to be inconsistent with the qualitative analysis and quantitative analysis, and the number of evaluation elements of the preliminary selection of indicators in this paper meets the basic requirements of the gray correlation analysis method, as shown in Table 1.

The indicators are deleted because of their low differentiation, while the market competition indicators are left because of their low relevance and low difficulty in obtaining, but from the qualitative point of view, because power supply enterprises have the characteristics of high monopoly and strong regulation by the state, the market competition of power enterprises is not fierce enough, and in the actual HR strategy decision, the market competition may not be considered, but more consideration should be given to the national policy, so the national policy indicators are finally retained. Therefore, the national policy indicators are retained, and the market competition indicators are deleted [18]. From the overall situation of employee turnover rate, it is maintained at a more reasonable level and lower than the average level of the industry. From the analysis of the turnover rate of employees in various categories, the turnover rate of key positions in $\mathrm{R} \& \mathrm{D}$, quality, functional management, and professional and technical personnel is higher than the industry average. The age structure of employees in general positions is older, the turnover rate is low, and redundancies are precipitated.

It is necessary to allocate human resources that match the strategic objectives with a strategic thinking and business perspective and support the implementation of the enterprise strategy through dynamic and synergistic functional activities. Strategic human resources create endogenous core competencies, cultivate unique and valuable core competencies that are difficult for competitors to imitate and rooted within the organization to gain sustainable competitive advantages, support the creation of core competencies, and are the fundamental source of core competencies and competitive advantages of the enterprise. With the advent of global economic integration and the era of the knowledge economy, the view that human resources have increasingly become the first resource of enterprises and the basis of competitive advantage has been generally recognized by management academics and business management practitioners. 
TABLE 1: Combined assessment values for the primary selection indicators.

\begin{tabular}{lcc}
\hline Primary selection indicators & Comprehensive evaluation value & Primary selection indicators \\
\hline$X 1$ & 30 & 49 \\
$X 2$ & 48 & 21 \\
$X 3$ & 23 & 38 \\
$X 4$ & 29 & 58 \\
$X 5$ & 78 & 39 \\
\hline
\end{tabular}

As organizations translate strategy into day-to-day business management activities, they must redefine organizational capabilities, HRM organizational structures, and practitioner competencies to align with HR transformation goals and positioning. The key role of HRM in creating a strategic competitive advantage for the organization is to build an organization that consistently produces better products than its competitors, creating organizational capabilities such as learning, market responsiveness, relationship coordination, and employee quality that can respond to change faster than competitors. Corporate human resource management can only truly demonstrate a competitive advantage in business if it has the organizational capability to outperform competitors' products and services, as shown in Figure 3.

The evaluation of alternative strategies for human resource strategies using reverse thinking requires the identification of criteria for an ideal human resource strategy. Then, the evaluation method that fits the object of study is selected for the study [19]. The HR strategy decision system is a gray system, and the method from gray system theory is selected to suit the problem. Constructing the HR strategy decision model through the above analysis requires first analysing the characteristics that the ideal HR strategy should have and selecting the evaluation indexes of HR strategy. Consistency means that the objectives of $\mathrm{HR}$ strategy are in line with the overall strategic objectives of the enterprise, which are mainly reflected in three aspects: the direction of the strategy, the degree of achievement, and the time of achievement. HR strategy is a substrategy formed based on the overall strategy. According to the principle that substrategies are subordinate to the overall strategy, their objectives should support the objectives of the overall strategy. Only by maintaining consistency can the human resources strategy properly guide human resources management activities and play a full role in enterprise management.

Support refers to the extent to which the core functions of human resources support the human resources strategy. The performance management function, the compensation management function, and the training management function together constitute the core functions of human resources. Among them, the performance management function is the central part, aiming to help employees improve their performance; the compensation management function aims to motivate people and solve the problem of "retaining" people; the training and development function aims to solve the problem of "nurturing" people. The support of each core function is the basis for the successful implementation of HR strategy [20]. Development refers to the smooth implementation of HR strategy and the sustainable development of the HR team, which is reflected in the optimization of HR quantity, improvement of quality, and reasonable structure. The human resource team is the main body of the implementation of human resource strategy, and a lower level of human resource team will hinder the implementation of human resource strategy, while a higher level of human resource strategy will cause excessive consumption of human resource team and weaken the overall strength. Therefore, the sustainability of the human resources workforce should not be ensured at the expense of its continuous development and the blind pursuit of short-term benefits.

\section{Results and Analysis}

4.1. RNN Algorithm Model Performance Analysis. After setting the values of each Green Cloud parameter, the simulation results include the total power consumption of the data centre as well as the power consumption data of each specific component, and the total power consumption data is chosen as the prediction object for the analysis. Each data set is a time series of 6000-time slot values. The algorithm in this paper uses both the convolution operation and the local join operation to take full advantage of both operations to learn higher-order abstract features on the data separately. The overall feature extraction property of convolution enables a holistic summary of the features for holistic discrimination, while the different representations of the local sensory domain of local connectivity enable a local characterization of the features for comparative discrimination. Then, a multilayer $\operatorname{ReLU}$ nonlinear activation function-based fully connected layer model is trained to map the sample marker space with the implicit layer feature space abstraction representation corresponding to the input data obtained from the previous convolution and local connection processing. Finally, the Sigmoid activation function fully connected layer is used as the output layer for the final classification process, the recommendation probabilities predicted by the two operations are obtained separately, and after averaging, the recommendation probabilities are converted into one of the "recommended/not recommended" dichotomous categories, which are presented as the final recommendation results, as shown in Figure 4.

The intermediate value pooling strategy is used because it can preserve the significant characteristics of the maximum features and obtain the data features about the wholeness by averaging. The data feature values obtained by this pooling strategy can consider the characterization of strong and weak features, which helps to reduce the training 


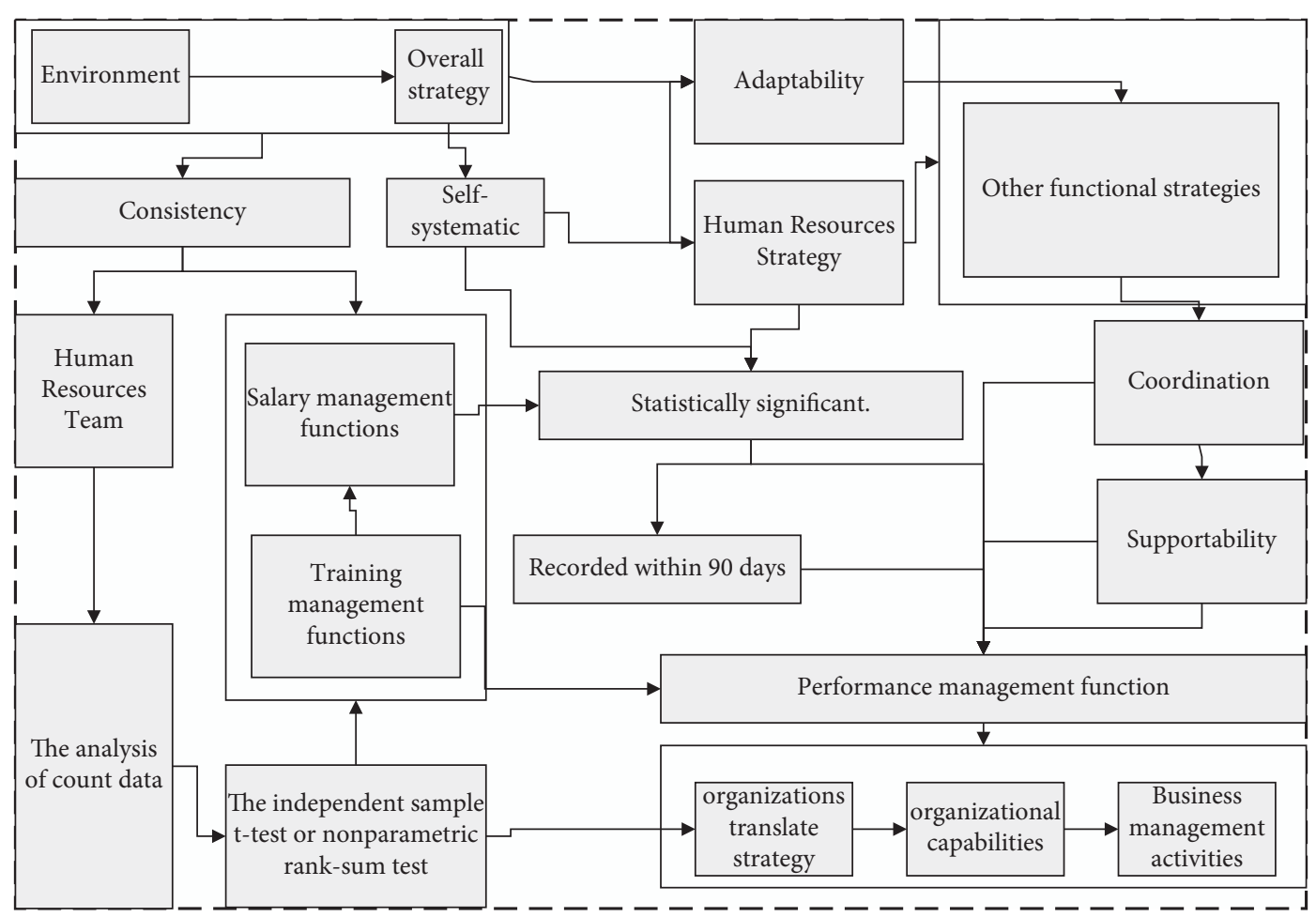

FIGURE 3: Diagram of the relationship between the ideal HR strategy and the influencing factors.

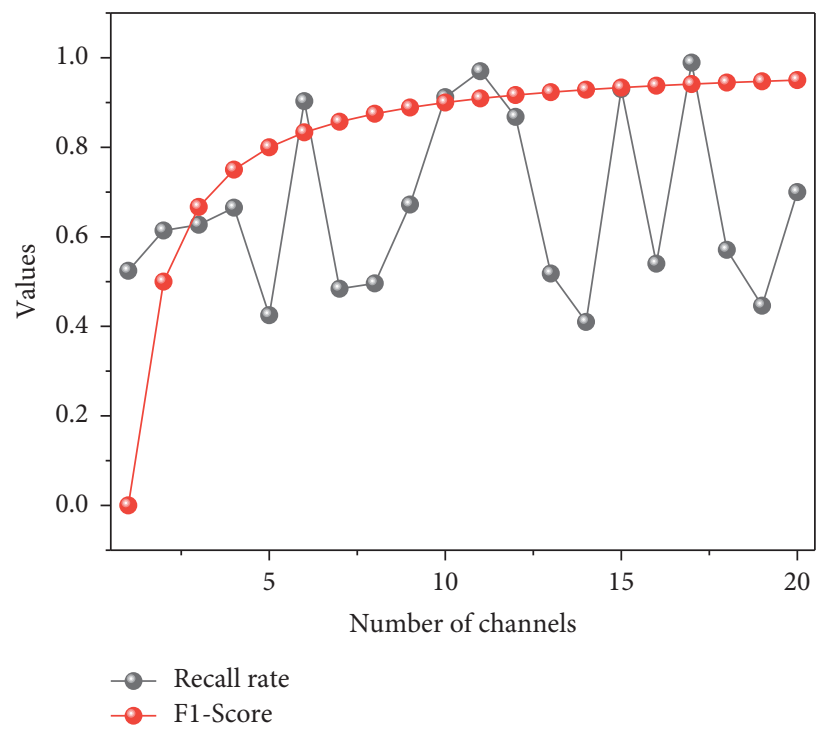

FIGURE 4: Experimental results of comparing the different number of channels.

error of the subsequent hierarchical model and improve the stability of the model, and the subsequent experimental results also confirm the advantages of the intermediate value pooling strategy. The optimization of hybrid multipooling strategy is adopted in pooling processing to improve the shortcomings of single pooling strategy, which can minimize the loss of features, retain the property representation of features themselves, and alleviate the problem of excessive feature information loss. More neighbourhood integration features are obtained while reasonably reducing the input data size of the subsequent hierarchical model, which maintains the ability to avoid the occurrence of overfitting phenomenon and enriches the diversity of training parameters of the subsequent hierarchical model to improve the recommendation performance of the model.

The results of multichannel convolution are all better than those of single-channel convolution, proving that the quality of recommendations can be improved by using multichannel convolution for feature extraction. When the number of channels is 2 , the recall F1-score is the highest 
and achieves better results than when the number of channels is 3 . The data types that need to be collected are classified according to the required type, and then multiple execution threads are allocated to execute data collection tasks in parallel on the distributed cloud cluster server for receiving data. It proves that when increasing the convolution channels until the optimal structure for feature extraction reached, further increasing the convolution channels will not be able to extract more useful representational features but will instead result in extracting too much useless information and affect the overall feature integration effect. Moreover, too many convolutional channels will also greatly increase the computational effort and require more computation time. Considering the overall algorithm performance and recommendation quality, the number of convolution channels is set to 2 in this paper, and the hybrid processing of the two-channel convolution method is performed.

In the training of the model, the learning rate has a great influence on the stability of the model. Too large a learning rate is likely to cause the correction of the learning parameters to become too large during training, making the model training difficult; however, too small a learning rate is likely to cause the model training time to become longer, and it is easy to fall into the local optimum problem. To obtain the optimal learning rate, this paper will conduct experimental comparisons of learning rates of different magnitudes, setting the learning rate to $0.007,0.05,0.1,0.2,0.3$, and 0.4 , respectively, to compare the effect by training loss and to compare the recommendation quality by $F 1$-score as the overall evaluation index, where the learning rate is initially set to 0.05 . The specific loss values of the experimental results are shown in Figure 5.

The model stabilizes at about 100 iterations, and the model reaches a state of basic convergence. From the comparison of the results, we can see that when the learning rate is set to 0.3 , the overall loss of the model is minimized and is in the best state. When the learning rate is less than 0.3 , the loss value is generally large, and the loss value decreases as the learning rate increases, which indicates that the learning rate less than 0.3 does not make the model optimal; when the learning rate is greater than 0.3 , the loss value is comparable to that of the learning rate of 0.3 when it is unstable, but the effect is less when it becomes stable, becomes difficult, and is not optimal for the model.

4.2. Simulation Results. This strategic factor drives the tendency for companies to adopt innovative talent management practices to retain and develop talent, rather than just acquire it, during economic downturns. Successful talent management systems integrate business planning and talent management processes and integrate talent management practices with other components of the HR management system. At the core of talent management that adopts a strategic model is the need to support business and structural change by planning the workforce to respond to those changes to achieve strategic corporate goals. This

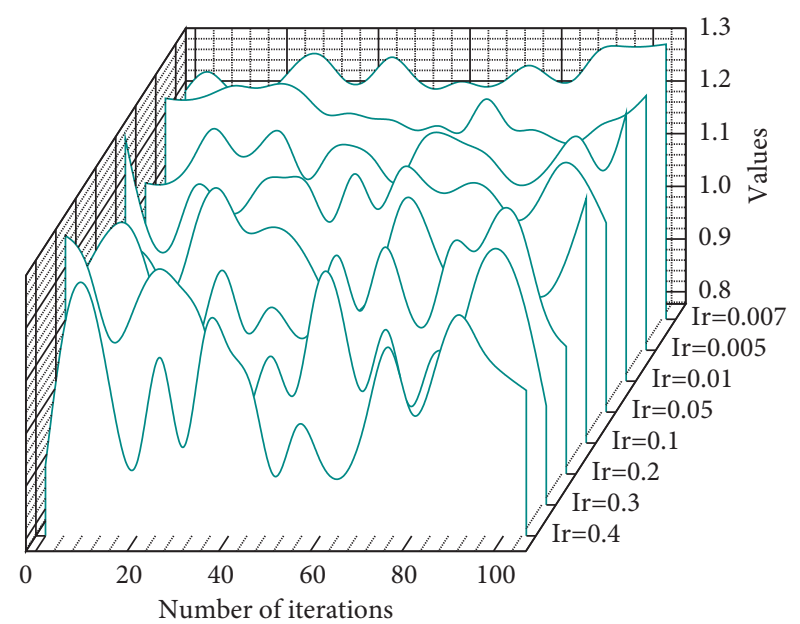

Figure 5: Comparison of loss for different learning rate parameters.

strategic motivation is also consistent with the assumptions of the resource-foundation view described earlier that talent resources, a competitive resource, can be developed and supported by a strategic talent management system, which can be said to be embedded in the organizational culture. Firms can circumvent the limitations imposed by institutional factors by recruiting and training employees who match the organizational culture.

Local companies are rooted in many years of history and cultural traditions, and this group-oriented cultural identity can weaken the positive impact of performance management, making it fundamentally difficult to change management styles that have long been embedded in the system, even if they have to accept new management models as a result of attracting investment from foreign companies, and their talent management tends to be short term, with management practices driven by more local politics to avoid positive conflict with traditional culture. The tactical model of talent management is more responsive to the current management need to make the most effective use of resources, develop leadership, retain staff, address skills shortages, attract new members, and redeploy staff to new roles, as shown in Figure 6.

The determination and selection of the overall sample should be considered when researching large sample data. Firstly, for the formal research, the people who are still selected are the head of the company, the middle and senior managers of the human resource management department, and the departmental scriptures to ensure the authenticity of the questionnaire data. Secondly, the questionnaires were distributed in the form of web-based electronic questionnaires and emails, and there were two main ways to collect data. Electronic questionnaires and emails were distributed through the connections of teachers, classmates, or family and friends. A human resource service company was commissioned to distribute the questionnaire among the relevant software park companies and service companies, as shown in Figure 7. 


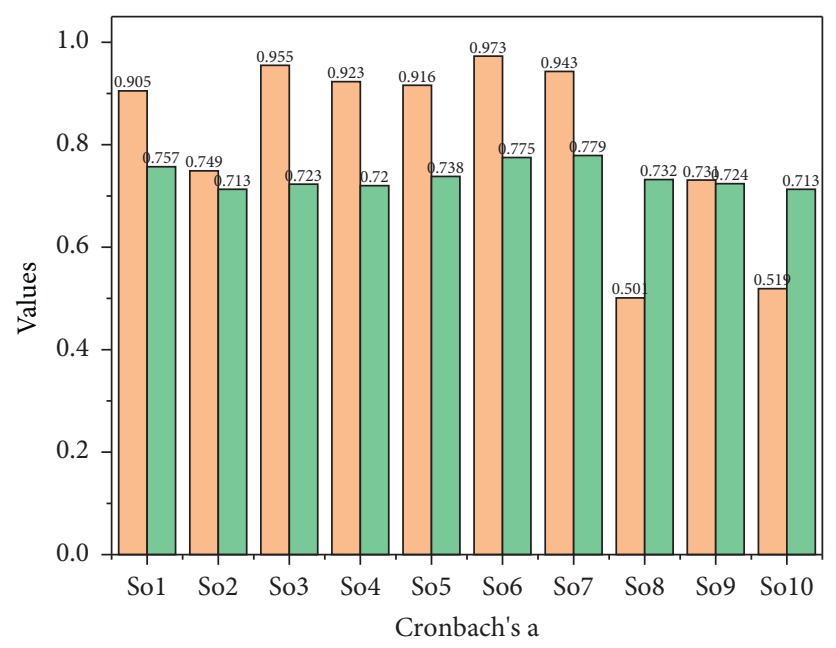

CITC

a

FIGURE 6: Organizational talent management implementation motivation scale CITC and reliability.

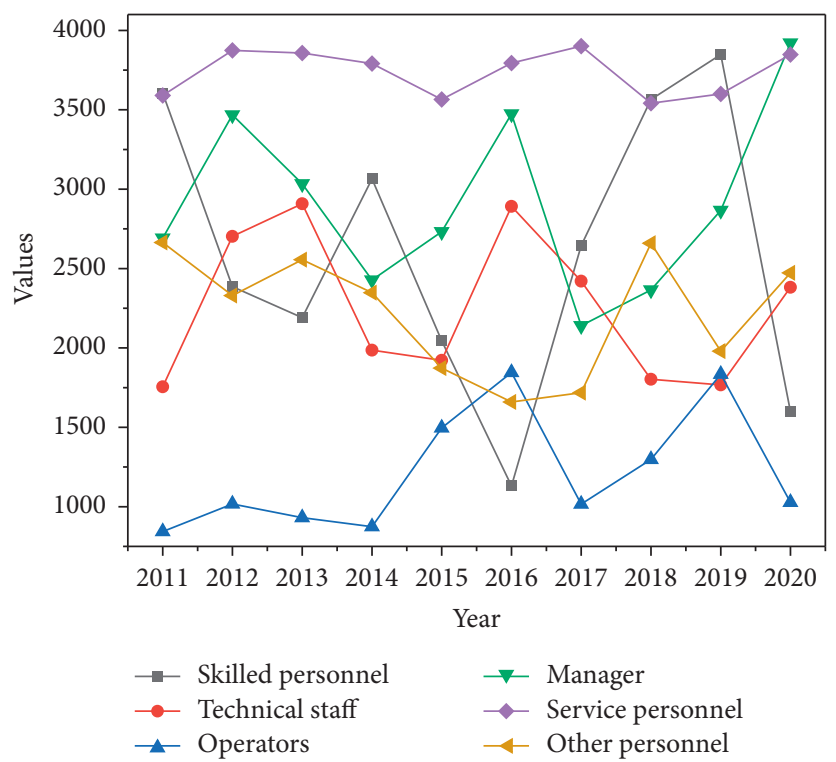

FIGURE 7: Simulation of changes to the personnel profile of a category.

The number of human resources is large, but there is a lack of technical personnel, the structure of human resources is unreasonable, and the level of personnel quality is uneven; the management of human resources has not formed a perfect mechanism, and the development and improvement of human resources management are constrained by traditional concepts and vested interests; the modern enterprise system is not perfect, and there are certain problems in the internal management of enterprises. When changes in the internal and external environment of enterprises become the norm, it is an inevitable requirement for position management to adapt to the new trend by classifying, analysing, and evaluating positions based on enterprise strategies and business processes, realizing dynamic management of position setting, changing, rising, and falling, and systematizing, standardizing, and institutionalizing. The main contents of traditional job management are job analysis, job description, and job evaluation, focusing on the management of specific jobs and the jobs within the organization, and the working relationships between jobs are relatively static and stable.

\section{Conclusion}

A common index system for strategic HR decision-making was established, and the factors affecting strategic HR decision-making include HR's workforce and management, as well as the micro-, macro-, and mesoenvironment in which it is located, and are characterized by 14 indicators such as HR quantity, HR quality, HR structure, enterprise strategy, level of economic development, and labour legal system. Compared to other industries, the electricity supply industry 
highlights the impact of national policies, which is determined by the nature of its industry. Relevant theories in this research area were compiled and reviewed, and a conceptual model of the strategic talent management system configuration was initially proposed based on the results of foreign literature. The initial identification of strategic talent management system configuration variables was made through qualitative research, the identified configuration variables were confirmed through theoretical analysis, and the conceptual model was revised on this basis. This was followed by a diagnosis, measurement, and evaluation of the internal fit and external fit between the construct variables. Finally, the possible diffusion and dissemination trends, evolution, and adjustment patterns of the strategic talent management system were analysed and predicted. The corporate strategic conformation variable is one of the important confirmation variables of the strategic talent management system. Through several revisions of the theoretical model, the alignment relationships of the three levels of strategic talent management system strategic constructs were confirmed.

\section{Data Availability}

The data used to support the findings of this study are available from the corresponding author upon request.

\section{Conflicts of Interest}

The authors declare that they have no conflicts of interest.

\section{Acknowledgments}

This work was supported by the Provincial Talent Cultivation Project, "Study on the Relationship between the Economic Behavior of Minority Farmers and the Ecological Environment in the Construction of Ecological Civilization," under KKSY201308035.

\section{References}

[1] R. H. Assaad and S. Fayek, "Predicting the price of crude oil and its fluctuations using computational econometrics: deep learning, LSTM, and convolutional neural networks," Econometric Research in Finance, vol. 6, no. 2, pp. 119-137, 2021.

[2] A. Hamza and N. Ben Yahia, "Heavy trucks with intelligent control of active suspension based on artificial neural networks," Proceedings of the Institution of Mechanical EngineersPart I: Journal of Systems \& Control Engineering, vol. 235, no. 6, pp. 952-969, 2021.

[3] X. Song, J. Man, S. Song, and Z. Ning, "Event-triggered synchronisation of Markovian reaction-diffusion inertial neural networks and its application in image encryption," IET Control Theory \& Applications, vol. 14, no. 18, pp. 2726-2740, 2020.

[4] H. R. Fogno Fotso, C. V. Aloyem Kazé, and G. D. Kenmoe, "Optimal input variables disposition of artificial neural networks models for enhancing time series forecasting accuracy," Applied Artificial Intelligence, vol. 34, no. 11, pp. 792-815, 2020.
[5] Q. Wang, L. Pan, K. Y. Lee, and Z. Wu, "Deep-learning modeling and control optimization framework for intelligent thermal power plants: a practice on superheated steam temperature," Korean Journal of Chemical Engineering, vol. 38, no. 10, pp. 1983-2002, 2021.

[6] K. Babojelić and L. Novacko, "Modelling of driver and pedestrian behaviour-a historical review," Promet-Traffic \& Transportation, vol. 32, no. 5, pp. 727-745, 2020.

[7] Y. Hua, X. Wang, Y. Li, P. Xu, and W. Xia, "Dynamic development of landslide susceptibility based on slope unit and deep neural networks," Landslides, vol. 18, no. 1, pp. 281-302, 2021.

[8] Z. Li, H. Guo, A. V. Barenji, W. M. Wang, Y. Guan, and G. Q. Huang, "A sustainable production capability evaluation mechanism based on blockchain, LSTM, analytic hierarchy process for supply chain network," International Journal of Production Research, vol. 58, no. 24, pp. 7399-7419, 2020.

[9] M. Benhaddi and J. Ouarzazi, "Multivariate time series forecasting with dilated residual convolutional neural networks for urban air quality prediction," Arabian Journal for Science and Engineering, vol. 46, no. 4, pp. 3423-3442, 2021.

[10] S. Grigorescu, B. Trasnea, T. Cocias, and G. Macesanu, "A survey of deep learning techniques for autonomous driving," Journal of Field Robotics, vol. 37, no. 3, pp. 362-386, 2020.

[11] M. Kohler, M. Vellasco, E. Silva, and K. Figueiredo, "SimProxy decision support system: a neural network proxy applied to reservoir and surface integrated optimization," IEEE Systems Journal, vol. 14, no. 4, pp. 5111-5120, 2020.

[12] T. Garg, M. Garg, O. P. Mahela, and A. R. Garg, "Convolutional neural networks with transfer learning for recognition of COVID-19: a comparative study of different approaches," Artificial Intelligence, vol. 1, no. 4, pp. 586-606, 2020.

[13] A. Sayghe, Y. Hu, I. Zografopoulos et al., "Survey of machine learning methods for detecting false data injection attacks in power systems," IET Smart Grid, vol. 3, no. 5, pp. 581-595, 2020.

[14] S. T. Abdulrazzaq, F. S. Omar, and M. A. Mustafa, "Decentralized security and data integrity of blockchain using deep learning techniques," Periodicals of Engineering and Natural Sciences, vol. 8, no. 3, pp. 1911-1923, 2020.

[15] A. Demidovskij and E. Babkin, "Integrated neurosymbolic decision support systems: problems and opportunities," Business Informatics, vol. 15, no. 3, pp. 7-23, 2021.

[16] K. B. Newhart, J. E. Goldman-Torres, D. E. Freedman, K. B. Wisdom, A. S. Hering, and T. Y. Cath, "Prediction of peracetic acid disinfection performance for secondary municipal wastewater treatment using artificial neural networks," ACS ES\&T Water, vol. 1, no. 2, pp. 328-338, 2020.

[17] J. L. Smith, "Advances in neural networks and potential for their application to steel metallurgy," Materials Science and Technology, vol. 36, no. 17, pp. 1805-1819, 2020.

[18] A. Massaro, G. Dipierro, A. Saponaro, and A. Galiano, "Data mining applied in food trade network," International Journal of Artificial Intelligence \& Applications, vol. 11, no. 2, pp. 15-35, 2020.

[19] A. M. Araba, Z. A. Memon, M. Alhawat, M. Ali, and A. Milad, "Estimation at completion in civil engineering projects: review of regression and soft computing models," KnowledgeBased Engineering and Sciences, vol. 2, no. 2, pp. 1-12, 2021.

[20] G. Dong, L. Cao, D. Yao, H. Li, and R. Lu, "Adaptive attitude control for multi-MUAV systems with output dead-zone and actuator fault," IEEE/CAA Journal of Automatica Sinica, vol. 8, no. 9, pp. 1567-1575, 2020. 